II.

\title{
Orthopädische Verbände.
}

Sammelreferat über die von Ende 1904 bis Anfang 1906 erschienenen Voröffentlichungen.

Voll

\author{
Dr. Wilhelm Becker, \\ Spozialarzt für Orthopädie in Bremen.
}

Mit 1 Abbildung im Text.

Von grösseren Monographien ist 1905 bei Enke in Stuttgart erschienen die Technik der Extensionsverbände von Bardenheuer und Grässner. Da das Werk bereits in dieser Zeitschrift eingehend besprochen wurde, so genügt an dieser Stelle der einfache Hinweis darauf.

Fangen wir mit dem Verbandmaterial an, so ist von mehr allgemein chirurgischem Interesse eine Arbeit von Brugger (Frankfurt a. M.) über die Verwendung der Spiritusverbände (1), in welcher deren Technik und Indikationen beschrieben und besonders auf die Feuersgefahr hingewiesen wird, die durch die Alkoholdämpfe beim Verbandwechsel entsteht.

Zelluloid als Wundverband empfieblt Wiener-Chicago (2). Er macht darauf aufmerksam, dass die bekannte Lösung von Zelluloid in Azeton ein äusserst bequemer Scbutzpanzer für viele kleinere und grössere Wunden ist. Dem zu diesem $Z$ weeke viel angewandten Kollodium ist es durch seine grössere Widerstandsfähigkeit weit überlegen. Die Tecbnik des Anlegens ist nach Verf. eine äusserst einfache; die des Abnehmens etc. wird nicht erwähnt, doch wird ein Abwaschen mit Azeton genügen. $\mathrm{Da}$ wohl jedem Orthopäden diese Lösung zur Verfügung steht, so dürfte ein Versuch nit derselben sich wohl empfehlen.

Nettel (Klinik v. Eiselsberg) schildert eine Modifikation bei der Herstellung der Gipshanfschiene (3). Die gekämmten Hanfstränge werden durch einen umgestülpten Trikotschlauch gezogen, dieser dann, nachdem das untere Ende zugebunden ist, mit Gipsbrei vollgegossen, und nach Verschluss des oberen Endes das ganze auf einem Tische durchgeknetet, so dass der Hanf überall gut durchtränkt ist. Der flach gestrichene Schlauch wird daun auf die gut eingefettete Extremität gelegt und mit einer Binde angewickelt; nach Erhärten desselben Abnahme und Zurechtschneiden.

Unter den Hilfsvorrichtungen sind zunächst von Kuhn (Kassel) Apparate zur Herstellung jeder Art von Extension (4) angegeben, deren Grundprinzip die Kombinierbarkeit einzelner an sich gleichartiger Teile ist, namentlich einfacher Stangen, Rollen und Klammern. Hierdurch lassen sich die verschiedensten mechanischen Effekte und Züge in allen möglichen Richtungen herstellen.

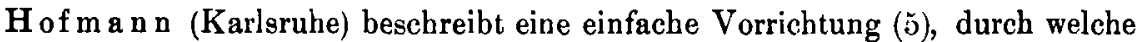
bei Extensionsverbänden der Längszug in queren Zug umgesetzt wird und so jeder Rollenträger und sämtliche Rollen vermieden werden. Als Vorteile werden gerühmt neben der denkbar einfachsten Improvisation Kostenersparnis in der Armenpraxis und Schonung kostbarer Bettstellen.

Neue Beckenstützen sind angegeben von $H$ ofmann (6), Gocht (7), Legal (8) und Finck (9). Die H of ma $n$ nsche Stütze besteht aus einem Stativ, einem horizontalen Lager und einem schrüg ansteigenden Dammwiderlager. Sie stellt im Prinzip nichts Neues dar, eignet sich auch weniger für Beckenverbände, kann dagegen sehr bequem im Bett beim Verbandwechsel gebraucht werden. G ocht hat seine Beckenstütze derartig konstruiert, dass sie nicht wie alle anderen 
von unten gestützt wird, sondern frei von oben herabbängt, daher auch beim Verbandanlegen nicht im Wege steht. Dasselbe erreicht Legal durch einen ausgespannten Filzstreifen, auf den der Patient gelagert wird; derselbe kommt mit in den Verband und wird später abgeschnitten. Die beste Beckenstütze, die auf einem ganz neuen Prinzip beruht, hat Finck angegeben. Er macht darauf aufmerksam, dass die bisherigen Stützen nur einen mehr oder weniger schmalen Streifen des Kreuzbeins angreifen, während die nicht unterstützten Nates wie zwei schlaffe Säcke herabhängen und dabei ungebührlich viel Raum im Verbande beanspruchen. Da nun aber für eine exakte Fixierung der Hüfte ein auch im Gesäss straff anliegender Verband unerlässlich ist, so hat $\mathrm{Finck}$ eine Beckenstütze konstruiert, auf welcher der Kranke wie auf zwei unterfassenden Händen ruht: präservative Kompression der Nates. Stellt man die beiden Blätter schräg nach aussen etwas auffallend - also in der Mitte höher - so hat man in der Tat für alle in Hyperextension des Hüftgelenkes (angeborene Hüftluxation!) anzulegenden Verbände eine Stütze, wie man sie sich besser nicht wünschen kann. Die Stütze selbst kann sich jeder nach seinem Verbandstsich modifizieren: das Prinzip der Kompression 'des Gesässes ist jedenfalls ebenso neu als vortrefflich.

Als Hilfsapparat für Becken- und Oberschenkelgipsverbände sei noch auf einen Urinfänger für Kinder (10) hingewiesen, den Grossmann (Frank. furt a. M.) angegeben hat. Jeder Arzt weis3, wie häufig Kinder, die sonst bettrein sind, nach angelegtem Gipsverbande Urin unter sich lassen. Für diese Fälle scheint der kleine Apparat gute Dienste zu leisten.

Die allgemeine Verbandtechnik ist wesentlich bereichert durch, einen von Gocht (Halle) angegebenen kombinierten Zug-Gipsverband. Bei Gipsverbänden, die unter stärkerer Extension eines Beines angelegt werden ist eine leider alltägliche Erscheinung ein oft schwerer Decubitus am Fussrücken und an der Ferse. Ganz natürlich: der Fuss hat ja allein den ganzen Gegendruck gegen die Extension auszuhalten. Diesem Übelstand hilft $\mathrm{Goch} \mathrm{t}$ ab, indem er den Gipsverband anlegt über einem Heftpflaster.Zugverband. Die Heftptlasterstreifen, die beiderseits oberhalb der Knöchelgegend aus dem Gipsverband herausragen, verschieben sich nach Aufhören des Zuges in den Gipsspalten nicht und werden um den Fussteil des Gipsverbandes gewickelt. Auf diese Weise wird der Fuss entlastet und der Druck auf das ganze Bein übertragen.

Legal (Breslau) beschreibt einen einfachen Lagerungs apparat fürdie untere Extremität (12). Es ist ein Gerüst aus Bandeisen, an dem eine Extensionsvorrichtung angebracht ist. Vor den gewöhnlichen Extensionsverbänden hat dies den Vorzug, dass der Patient zwar liegen muss, aber an keine bestimmte Cagerstatt gebunden ist (Kriegstransport!), dass jede beliebige Stelle der Extremität leicht zugängig ist uud dass der Patient bei der Defäkation seinen Unterkörper leicht hochheben kann.

Mehr in das chirurgische Each greift ein Verfahren über, das Frey (Geisingen) als Gewichtsextension der $\mathrm{Haut}$ (13) beschreibt. Zur Entlastung der Hautnähte hat Verf. bei einer Amputationswunde mit etwas zu kurz geratener Hautmanschette mittelst Heftpflasterstreifen die Haut der Nachbarschaft herangezogen und so eine gute Entspannung der Nähte erzielt.

Wir wenden uns jetzt zur speziellen Verbandstechnik. Natürlich können hier im engen Rahmen eines Sammelreferates nicht alle in obigem Zeitraum angegebenen Verbände und Verbandmodifikationen Platz finden, zumal da viele Spezialverbände mehr in das betr. orthopädische Spezialkapitel gehören, z. B. Klumpfussverbände unter Klumpfuss. Wir glaubten uns daher auf einige Verbände von mehr allgemeinem Interesse beschränken zu sollen.

Zur Korsettbehandlung der $R$ ückgratsverkrümmungen hat Gerson (Berlin) (14) einen abnehmbaren, vorn und hinten aufgeschnittenen und durch Gummischnüren zusammengehaltenen Gipsverband beschrieben, der allerhand Vor- 
teile haben soll. Von Hahn (Maiuz) ist ein Extensionskopfträger (15) angegeben, der aus einer Rückenstange, einem Kopfteil und einer in einer Hülse steckenden Spiralfeder besteht. Er soll eine dauernde Extension des Kopfes bewirken, was jedoch undenkbar ist, da er am Kinn angreift. Verf. hat ihn zur Behandlung der Skoliose und der Spondylitis benutzt.

Zur Technik des Gipsbettes (16) verwendet $\mathrm{H}$ a udek (Wien) keine eigentlichen Binden, sondern entsprechend abgeschnittene Gazestücke, die gut mit Gips eingestrichen und in Lagen von je drei Stück lose zusammengerollt werden. Sie sollen eine möglichst rasche und an der Innenseite vollkommen glatte Anfertigung ermöglichen.

Über Gipsverbandbehandlung bei Ischias (17) berichtet Cramer (Köln). Ein Verband des erkrankten Beines inkl. Fusses - ein Einbeziehen der Hüfte ist meist unnötig - in leichter Flexion, Abduktion und Aussenrotation der Hüfte, sowie geringere Flexion des Knies beseitigen fast stets den heftigen Schmerz, was durch Entlastung des Ischiadicus von Druck und Zug, sowie durch Herabsetzung der venösen Hyperämie erklärt wird.

Kofmann (Odessa) legt zur ambulanten Behandlung von Oberschenkelfrakturen (18) einen Gipsverband ähnlich dem Lsorenz schen Coxitisverband an, der nach unten bis über das Kniegelenk reicht und einen Gehbügel trägt. Gegen diesen zieht eine Spannlasche den Fuss für die ersten Tage und für nachts. Der Verband wird angelegt über einem Trikotschlauch, der bis zur Nabelhöhe geht; gepolstert werden nur Christae, Spinae, Trochanter, Tuber und Patella.

Für Oberschenkelbrüche von Säuglinger ist ein sehr zweck. mässiges Streckbett von Landau (Berlin) angegeben worden (19). An einem

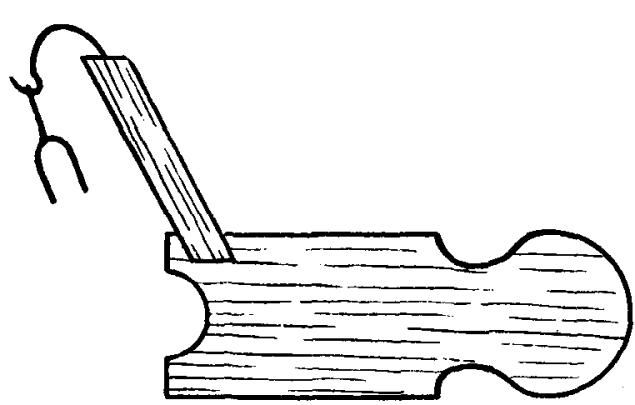
für Kopf und Rumpf von jedem Tischler leicht zurecht $\mathrm{zu}$ schneidenden Brett ragt in schräger Richtung nach obenaussen für das gebrochene Bein eine Art von Galgenpfosten mit Querbügel hervor. Zur Anwendung wird am kranken Beinchen zunächst ein Streckverband angelegt und dann das Kind auf das gepolsterte Brett mit Binden angewickelt, so dass das Gesäss mit dem Rande des Brettes abschneidet und die Hüftbindentouren als Kontraextension dienen. Darauf wird der Bügel des Streckbandes in den mit Gummizug versehenen Querriegel des Pfostens eingebängt.

Der Vorzug vor der vertikalen Suspension des Beines im Bette nach Schede besteht darin, dass der Säugling mit diesem kleinen Apparat jederzeit aufgenommen, gereinigt und an die Brust gelegt werden kann, ohne dass die extendierende Fixation auch nur für einen Moment unterbrochen wird.

Zum Schluss sei noch kurz eines Klumpfussverbandes von Nobe (Braunschweig) gedacht, nicht, weil ihn Ref. für praktisch hält, sondern weil er in keiner orthopädischen Fachzeitschrift (20) veröffentlich $t$ ist. Bei der Anlegung wird die von Sprengel angegebene Blechsoble auf ein kleines Stativ gestellt und kommt mit in den Verband, wodurch vermieden wird, dass während der Anlegung der Fuss, gegen den der Unterschenkel als langer Hebelarm benutzt wird, von der Tischplatte aufgehoben zu werden braucht.

\section{Literaturnachweis.}

1. Deutsche med. Woch. 1905. Nr. 7. - 2. Zentralbl. f. Chirurg. 1905. Nr. 43. 3. Wiener klin. Woch. 1904. Nr. 48. - 4. Münch. med. Woch. 1905. Nr. 23. - 5. Ibidem 
1906. Nr. 6. - 6. Zentralbl. f. Chir. 1905. Nr. 10. - 7. H of fa, Zeitschr. Bd. XIII. 8. Ibidem, Bd. XIV. - 9. Zentralbl. f. Chir. 1905. Nr. 39. - 10. Münch. med. Woch. 1905. Nr. 50. - 11. H off a, Zeitsehr. Bd. XIII. - 12. Ibidem, Bd. XIV. - 13. Munch. med. Woch. 1905. Nr. 33. - 14. H off a, Zeitschr. Bd. XIV. - 15. Münch. med. Wocb. 1905. Nr. 30. - 16. Zentralbl. f. Chir. 1905. Nr. 7. - 17. Ho f fa, Zeitschr. Bd. XIV. - 18. Zentralbl. f. Chir. 1904, Nr. 49. - 19. Deutsche med. Woch. 1904. Nr. 50. - 20. Zentralbl. f. Chir. 1905. Nr. 12.

III.

\title{
Belgische Literatur des Jahres 1905.
}

\author{
Bericht von Dr. A, van Haelst in Gand.
}

Im Laufe des Jahres 1905 hat sich die wissenschaftliche Tätigkeit in Belgien fast ganz auf die verschiedenen Kongresse gelegentlich der Weltausstellung zu Lüttich konzentriert. Es wird zur Nachlese wenig übrig bleiben.

Unter den Veröffentlichungen über angeborene Anomalien heben wir zunächst hervor einen Fall von Ektopie des linken Hodens mit Fehlen des Gliedes, des Nebenhodens, der Samenbläschen, der entsprechenden Hälfte der Vorsteherdrüse und des vas deferens. Ch. Beyer ${ }^{1}$ ), der diese äusserst seltene Missbildung gelegentlich der Operation eines Leistenbruches konstatieren konnte, geht näher auf die Ätiologie der Missbildung ein.

N. Moeller ${ }^{2}$ ) berichtet über einen Fall von Makrodaktylie. Es handelt sich um einen Fall von Elephantiasis vera des kleinen Fingers der rechten Hand bei einem jungen Mann. Die Vergrösserung des Umfangs war verursacht durch weiches Gewebe von fettiger Konsistenz. Die Knochen sind normal mit Ausnahme der Spitze des dritten Fingergliedes, welche hypertrophisch ist. Der Verfasser hat den Fall mit vollem Erfolg behandelt durch Exstirpation der gewucherten Partien und der hypertrophischen Phalanx.

Die Polydaktylie ist Gegenstand von zwei kleinen Arbeiten von N. Moelle $\mathbf{r}^{3}$ ) und von $D \mathrm{uhat}^{4}$ ).

Zwei Verfasser berichten über Beobachtungen von Imperforation des Anus mit Einmündung des Mastdarms in die Vulva. (N. Moeller und A. van Ha el s t).

Gommaerts ${ }^{5}$ ) berichtet an der Hand klinischer Fälle über die verschiedenen Hilfsmittel einer rationellen Gymnastik gegen Störungen seitens der Atmungswege.

Frau Derscheid-Dehourt ${ }^{6}$ ) veröffentlichte eine Abhandlung über Pottsche Krankheit, in der sie eine Vorrichtung zur Erhaltung des Redressements mittelst einer federnden Pelotte beschreibt. Die narkotisierten Patienten werden in einem einfachen Eisenrahmen so in lordotischer Stellung gelegt, dass die Partien oberhalb und unterhalb des Gibbus gut unterstützt sind. Dann folgt Extension und mässig starke Redression, Anlegen der federnden Pelotte und Gipsverband. Die weitere Behandlung besteht in Immobilisation in Bettlage und dauert 8-12 Monate. Später tragen die Kinder ein Stützkorsett. Der in den Gipsverband eingeschlossene Apparat besteht aus einer starken, gebogenen Feder aus Stahl, an den Enden befestigt auf zwei Plättchen. Der konvexe Teil trägt eine leicht ausgehöhlte, stark gepolsterte Pelotte. Die Erfolge waren recht gute?).

Die Sehnentransplantation, verworfen von den einen, in den Himmel erhoben von den andern, bildet den Gegenstand einer kritischen Studie von 